\title{
Genome-wide analysis of alternative splicing in Chlamydomonas reinhardtii
}

\author{
Adam Labadorf ${ }^{1}$, Alicia Link², Mark F Rogers ${ }^{1}$, Julie Thomas², Anireddy SN Reddy²* Asa Ben-Hur ${ }^{{ }^{*}}$
}

\begin{abstract}
Background: Genome-wide computational analysis of alternative splicing (AS) in several flowering plants has revealed that pre-mRNAs from about 30\% of genes undergo AS. Chlamydomonas, a simple unicellular green alga, is part of the lineage that includes land plants. However, it diverged from land plants about one billion years ago. Hence, it serves as a good model system to study alternative splicing in early photosynthetic eukaryotes, to obtain insights into the evolution of this process in plants, and to compare splicing in simple unicellular photosynthetic and non-photosynthetic eukaryotes. We performed a global analysis of alternative splicing in Chlamydomonas reinhardtii using its recently completed genome sequence and all available ESTs and cDNAs.

Results: Our analysis of AS using BLAT and a modified version of the Sircah tool revealed AS of 498 transcriptional units with 611 events, representing about 3\% of the total number of genes. As in land plants, intron retention is the most prevalent form of AS. Retained introns and skipped exons tend to be shorter than their counterparts in constitutively spliced genes. The splice site signals in all types of AS events are weaker than those in constitutively spliced genes. Furthermore, in alternatively spliced genes, the prevalent splice form has a stronger splice site signal than the non-prevalent form. Analysis of constitutively spliced introns revealed an over-abundance of motifs with simple repetitive elements in comparison to introns involved in intron retention. In almost all cases, AS results in a truncated ORF, leading to a coding sequence that is around 50\% shorter than the prevalent splice form. Using RTPCR we verified AS of two genes and show that they produce more isoforms than indicated by EST data. All CDNA/EST alignments and splice graphs are provided in a website at http://combi.cs.colostate.edu/as/chlamy.

Conclusions: The extent of AS in Chlamydomonas that we observed is much smaller than observed in land plants, but is much higher than in simple unicellular heterotrophic eukaryotes. The percentage of different alternative splicing events is similar to flowering plants. Prevalence of constitutive and alternative splicing in Chlamydomonas, together with its simplicity, many available public resources, and well developed genetic and molecular tools for this organism make it an excellent model system to elucidate the mechanisms involved in regulated splicing in photosynthetic eukaryotes.
\end{abstract}

\section{Background}

The coding regions (exons) of most eukaryotic genes are interrupted by non-coding sequences (introns). The intronic sequences from primary transcripts (also called precursor-mRNAs or pre-mRNAs) are removed and the exons are spliced to generate functional mRNAs [1]. In many organisms, pre-mRNAs are alternatively spliced to generate multiple mRNAs from a single gene [1]. It is

\footnotetext{
* Correspondence: reddy@colostate.edu; asa@cs.colostate.edu

'Computer Science Department, Colorado State University, Fort Collins, CO, USA

${ }^{2}$ Department of Biology and Program in Molecular Plant Biology, Colorado State University, Fort Collins, CO, USA
}

becoming clear that alternative splicing generates distinct proteins with altered functions from a limited set of genes [2-5]. The effects of alternative splicing on proteins include production of protein isoforms with loss or gain of function, altered subcellular localization, protein stability and/or posttranslational modifications [1,3]. Furthermore, alternative splicing plays a role in regulation of gene expression through processes such as regulated unproductive splicing and translation (RUST) and mRNA recruitment $[6,7]$. Alternative splicing is also implicated in evolution of organisms [8]. The availability of the complete genome sequences of many multicellular eukaryotic organisms and large sets of full-length 
cDNAs and ESTs has permitted comprehensive analysis of alternative splicing. More recently, global analysis of alternative splicing has also been performed using splicing sensitive mircroarrays and new generation sequencing technologies $[5,8,9]$. These analyses have shown that alternative splicing is highly prevalent in multicellular eukaryotes. In humans, 95\% of multiexon genes undergo alternative splicing resulting in the generation of two or more transcripts from a single gene [5]. Analysis of alternative splicing in flowering plants by aligning the available cDNAs/ESTs to genome sequences has shown that pre-mRNAs from $\sim 30 \%$ of genes are alternatively spliced [10,11]. Alternative splicing in some specific gene families such as genes encoding serine/ arginine-rich proteins is extensive, resulting in a fivefold increase in transcriptome complexity [12,13]. In addition, stresses have been shown to dramatically alter the splicing pattern of many plant genes [3,12-16]. In mammalian systems, exon skipping is most prevalent, whereas in flowering plants up to $55 \%$ of alternative splicing events are intron retention $[3,10,11,17]$. It is suggested that the variations in frequencies of different types of alternative splicing events between plant and non-plant systems reflect the differences in gene architecture and pre-mRNA splicing between these organisms [3,11]. In contrast to multicellular organisms, very little is known about the prevalence and types of alternative splicing in simple unicellular photosynthetic eukaryotes from which land plants have evolved. Recent completion of the Chlamydomonas genome and the availability of a fairly large number of ESTs [18-20] permit global analysis of post-transcriptional events including alternative splicing in a unicellular photosynthetic eukaryote.

Chlamydomonas shares many features with cells of more complex eukaryotic plants and animals. Chlamydomonas, like land plants, is an autotroph and contains a chloroplast. Furthermore, like animals, it can grow as a heterotroph and is mobile. Chlamydomonas diverged from land plants about one billion years ago [18]. About $93 \%$ of the $120 \mathrm{Mb}$ genome of Chlamydomonas reinhardtii has been sequenced [18,19]. Gene models in the latest version (v4) of the Chlamydomonas genome sequence predict 16,709 protein-coding genes and about half of these gene predictions have cDNA/EST support. Analysis of the Chlamydomonas genome revealed that it contains many genes that are specific to both plant and animal lineages, reflecting its unique position in evolution [18]. Because of the many advantages Chlamydomonas offers, it is considered to be the "green yeast" for studying various eukaryotic cellular processes [21]. For over five decades, Chlamydomonas has been used as a model system to study many aspects of photosynthesis, structure and function of flagella, and a variety of other biological processes. More recently, it is being used to investigate mechanisms that regulate biofuels production [22]. Analysis of alternative splicing in Chlamydomonas allows comparison of alternative splicing between simple unicellular photosynthetic eukaryotes and highly evolved flowering plants.

Furthermore, this will also aid in understanding how alternative splicing has evolved during the evolution of land plants. Hence, we have performed a comprehensive analysis of alternative splicing in Chlamydomonas reinhardtii. We have used the BLAT tool [23] and a modified version of the Sircah software for the detection and visualization of alternative splicing [24]. Detailed results, including alignments and splice graphs for each cluster exhibiting alternative splicing are available on our "Chlamydomonas AS" site http://combi.cs.colostate.edu/ as/chlamy/. Our results show that alternative splicing is prevalent in Chlamydomonas, although the extent of it is less than in land plants. The relative frequency of different splicing events in Chlamydomonas is very similar to higher plants.

\section{Results and Discussion \\ Properties of introns}

Unlike other unicellular eukaryotes (e.g. yeast), the vast majority of genes $(\sim 88 \%)$ in Chlamydomonas have introns (see Table S1 in the Additional file 1 for a comparison of the properties of the Chlamydomonas genome with humans and Arabidopsis). The percentage of intron-containing genes in Chlamydomonas is higher than in plants and humans.

Previous comparative studies on gene structure in flowering plants and animals have revealed a number of significant differences in their gene architecture [25,26]. For example, land plant genes are shorter than animal genes with fewer exons and shorter introns [3].

Furthermore, plant introns are rich in $\mathrm{T}$ or $\mathrm{T} / \mathrm{A}$, which is necessary for the recognition of splice sites and efficient splicing of pre-mRNAs $[25,26]$. Chlamydomonas shares both plant and animal features in its gene architecture. The average number of introns in Chlamydomonas is similar to humans. However, the median size of exons (132 bases) and introns (232 nucleotides) is similar to flowering plants. The GC content of Chlamydomonas is $64 \%$, which is significantly higher than the GC content of multi-cellular organisms. Introns in protein coding genes of metazoans have four signals that are necessary for accurate splicing of pre-mRNAs. These include two consensus sequences at the 5' and 3' splice sites with conserved GT and AG dinucleotides, respectively, a polypyrimidine tract at the 3 ' end of the intron, and a branch point located about 17-40 nucleotides upstream of the 3' splice site [1]. However, in land-plants the branch point is not obvious; the 3 ' end 
of plant introns are rich in T nucleotides [25]. Although the 5' and 3' splice sites in Chlamydomonas introns are similar to land plants and humans (Figure S5 in Additional file 1) the 3 ' end of introns in Chlamydomonas is enriched in $\mathrm{C}$ in place of a polyprimidine tract (Figure $\mathrm{S} 6$ in Additional file 1).

\section{Extent and types of alternative splicing}

We developed a pipeline for detection and visualization of alternative splicing in Chlamydomonas based on EST-to-genome alignments using BLAT [23], and a modified version of the Sircah alternative splicing detection software [24]. Details are found in the Methods section and Additional file 1. For EST data we used a recently constructed EST dataset containing 252,484 ESTs processed using cDNA termini to anchor transcripts to their correct positions in the genome [20]. ESTs were aligned to the genome and grouped into clusters that overlap in their genomic coordinates and occur on the same strand. Our alignment and alternative splicing detection pipeline resulted in 498 clusters that show 611 alternative splicing events. The alternative splicing events in each cluster are summarized with splice graphs [27]. Example splice graphs are shown in Figure 1. A companion website provides visualization of the EST alignments and splice graphs for each cluster showing alternative splicing, as well as access to the alignments themselves and additional information (see http://combi.cs.colostate.edu/as/chlamy/). Of the clusters that show alternative splicing, 484 were associated with predicted genes in version 4.0 of the Chlamydomonas genome [28].

We classified all observed alternative splicing events into the following five groups: Intron Retention (IR), Alternative 5' splice site (Alt5'), Alternative 3' splice site (Alt3'), events where both the $5^{\prime}$ and the $3^{\prime}$ end of an intron are alternatively spliced (AltB), and Exon Skipping (ES). The relative frequency of the various types of alternative splicing events is very similar to those observed in other plant species, with intron retention making up almost half of the events. Detailed statistics are provided in Table 1.

\section{Splice site strength}

We compared the splice site strength of the 5' and 3' splice sites in all types of alternative splicing events to those of constitutively spliced genes using the protocol described in [29]. Consistent with observations in other organisms [29], splice sites that participate in alternative splicing are weaker than constitutive splice sites, and all the differences are statisitically significant (see Table 2). The most significant difference is found at the 3' splice site of Alt3' events. In each alternative splicing event, we identified the prevalent splice form as the one supported by the largest number of ESTs. We observed that in the case of Alt5' and Alt3' events the splice sites for the non-prevalent splice forms are weaker than those of the prevalent splice form; the latter are weaker than those observed in constitutive splicing (see Table 3 and Figure 2 ). These differences are also highly statistically significant.

\section{Length and GC content of retained introns and skipped exons}

Alternatively spliced introns and exons in multicellular organisms were shown to have different length and nucleotide composition than their constitutively spliced counterparts $[11,30]$. We compared the length of retained introns and skipped exons with those that did not exhibit alternative splicing. This analysis revealed that retained introns are shorter than those that did not exhibit alternative splicing. The median size of retained introns is $127 \mathrm{bp}$ compared to a median size of $232 \mathrm{bp}$ in constitutively spliced introns. The difference is more pronounced in Chlamydomonas than in Arabidopsis, where median sizes are $93 \mathrm{bp}$ compared to $100 \mathrm{bp}$ [30]. Skipped exons are shorter than exons that are not known to exhibit alternative splicing with a median size of $84 \mathrm{bp}$ compared to a median size of $132 \mathrm{bp}$ in constitutively spliced exons.

In land plants, introns have high AT content, whereas exons are GC rich. Subsequently, a high percentage of $\mathrm{A} / \mathrm{T}$ or $\mathrm{T}$ was reported to be important for efficient splicing of introns in flowering plants. Proteins that bind to U-rich stretches in pre-mRNA have been reported in plants [26,31]. In Chlamydomonas, we found that retained introns have a GC content of $57 \%$ as compared to $62 \%$ for constitutive introns. Furthermore, short, inframe introns have an even lower GC content (56\%). Similarly, skipped exons have a lower GC content as compared to constitutive exons (63\% versus $66 \%$ ). All these differences are highly statistically significant $(t$ tests yielded $p$ values of $4.1475 \mathrm{e}-35,2.5601 \mathrm{e}-06$, and 0.0031, respectively). In Arabidopsis, the opposite trend is observed; retained introns have a higher GC content [30].

\section{Impact of alternative splicing on predicted proteins}

Alternative splicing often results in the occurrence of a premature termination codon (PTC) [1,6]. Transcripts with PTCs are potential targets for degradation through non-sense mediated mRNA decay (NMD) [32,33]. Several recent studies suggest that the alternative splicing of pre-mRNAs is coupled to mRNA degradation through regulated unproductive splicing and translation, (RUST) $[6,7,34]$. To analyze predicted proteins, we focused on clusters that have full-length cDNAs and a single alternative splicing event so that we can predict 

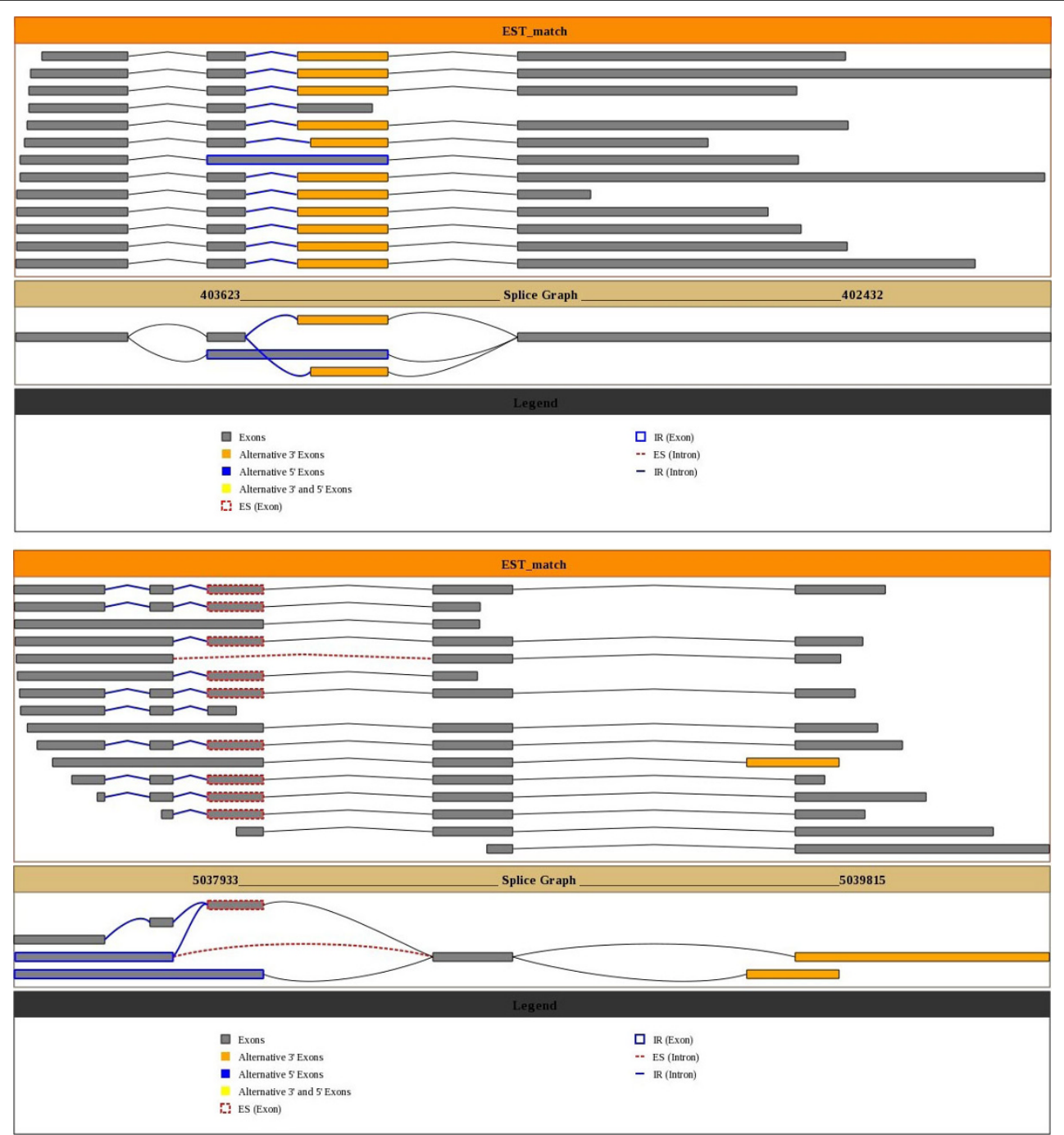

Figure 1 Example splice graph. Shown are two splice graphs along with the relevant EST evidence for the gene fgenesh2_pg. C_scaffold_39000087, which exhibits intron retention and Alt3' (top), and for the gene estExt_fgenesh2_kg.C_380020, which exhibits intron retention, exon skipping, and Alt3' (bottom). Figures were generated by Sircah as part of our pipeline.

the effect of alternative splicing on the resulting protein. Out of the 498 clusters showing AS, 483 correspond to annotated genes. Of these, 416 have published start codons, and 77 had a single AS event, and include a stop codon within a full-length EST. In 76 out of these 77 clusters, whenever alternative splicing occurred in the coding region, the non-prevalent splice form led to a shorter protein because of a PTC, resulting in a protein that is around $50 \%$ shorter (see Table 4). In Arabidopsis $78 \%$ of alternative splicing events occur in the coding region and about $50 \%$ of these have a PTC [11]. It has been shown in plants that transcripts with a PTC undergo NMD, and some of the components involved in NMD have been reported in plants [34-37]. Interestingly, the predicted proteome of Chlamydomonas contains components of NMD such as UPF3 and exon- junction complex proteins, suggesting that the NMD might play a role in the regulation of gene expression.

\section{Alternative splicing motifs}

Since introns have four loosely conserved signals, it is thought that other sequences may be involved in regulated splicing. In metazoans and land plants, protein factors such as SR proteins and hnRNPs have been shown to regulate splicing by binding to such splicing regulatory elements either in exons or introns and to enhance or prevent the usage of a splice site $[38,39]$. We performed a motif analysis of retained introns in comparison to constitutive introns using the DME program [40] (see details in the Additional file 1). While we didn't find statistically significant motifs in retained introns, constitutively spliced introns consistently produced such 
Table 1 The prevalence of different types of alternative splicing events.

\begin{tabular}{cccc}
\hline & Chlamydomonas & Arabidopsis & Rice \\
\hline IR & $305(50.0 \%)$ & $4635(56.1 \%)$ & $7774(53.5 \%)$ \\
\hline ES & $73(11.9 \%)$ & $666(8.1 \%)$ & $2004(13.8 \%)$ \\
Alt5' $^{\prime}$ & $71(11.6 \%)$ & $845(10.2 \%)$ & $1642(11.3 \%)$ \\
Alt3 $^{\prime}$ & $158(25.8 \%)$ & $1810(26.0 \%)$ & $2201(15.5 \%)$ \\
AltB & $4(0.7 \%)$ & $308(3.7 \%)$ & $921(6.3 \%)$ \\
Total & 611 & 8264 & 14542 \\
\hline
\end{tabular}

This table shows the number and frequency of each type of alternative splicing. Percentage of the total number of events is shown in parentheses. The statistics for Arabidopsis and rice are from [11].

motifs. All the motifs were tandem repeats of di-nucleotides or tri-nucleotides. The consensus sequence for the top scoring motif was TGCTGCTG. A complete list of motifs with their associated p-values is presented in Table S4 in Additional file 1. Simple repetitive elements have been shown to bind splicing regulatory proteins such as SRs and hnRNPs and contribute to regulated splicing [41]. In Chlamydomonas there are several SR and hnRNP proteins that share significant sequence similarity with splicing regulators in multicellular organisms.

\section{Experimental verification of alternative splicing}

For experimental verification, we chose two of the clusters corresponding to ornithine decarboxylase 1 (ODC1, gene ID: OVA2_SAN_estEXT_fgenesh2_kg.C_340012) and asparagine synthase (ASyn, gene ID: estExt_fgenesh2_kg.C_280076), and performed reverse transcription PCR (RT-PCR). Amplification of DNAse-treated RNA with primers corresponding to these genes did not yield any products (Figure 3A), suggesting no DNA

Table 2 Splice site strength in alternative and constitutive splicing.

\begin{tabular}{lcccc}
\hline Event & \multicolumn{2}{c}{$\mathbf{5}^{\prime}$ site } & \multicolumn{2}{c}{$\mathbf{3}^{\prime}$ site } \\
\hline & $\begin{array}{l}\text { motif } \\
\text { score }\end{array}$ & $p$-value & $\begin{array}{c}\text { motif } \\
\text { score }\end{array}$ & $p$-value \\
\hline $\begin{array}{lcccc}\text { Intron } \\
\text { Retention }\end{array}$ & 7.790 & $7.492(6.258 \mathrm{e}-44)$ & 7.165 & $6.925(8.734 \mathrm{e}-11)$ \\
\hline Exon & 6.701 & $6.156(1.465 \mathrm{e}-09)$ & 7.735 & $6.921(4.402 \mathrm{e}-12)$ \\
Skipping & & & & \\
\hline Alt 5' & 7.304 & $6.373(7.175 \mathrm{e}-20)$ & 7.448 & $7.097(0.0517)$ \\
\hline Alt 3' & 8.594 & $8.434(0.00176)$ & 5.460 & $3.478(1.686 \mathrm{e}-80)$ \\
\hline Constitutive & 8.822 & $\mathrm{~N} / \mathrm{A}$ & 7.574 & N/A \\
\hline Average splice & sccos & & \\
\hline
\end{tabular}

Average splice site scores and $p$-values for alternative splicing events and constitutive splicing are shown here. All scores are computed with respect to the splice site motif of the constitutive splice form, following the protocol used in [29]. In all cases, the scores for the alternatively spliced form are lower than for constitutive splicing. The $p$-values are based on a comparison of the scores for each type of alternative splicing event with the scores for constitutive splicing, and are computed using the Wilcoxon signed-ranks test. Except for the case of exon skipping, the $5^{\prime}$ and $3^{\prime}$ sites refer to the splice sites of an excised intron. In exon skipping the $5^{\prime}$ and $3^{\prime}$ sites are the splice sites flanking the skipped exon. contamination in our RNA. Our RT-PCR analysis with primers corresponding to the first and last coding exons showed six splice variants with ODC1 (Figure 3B) and two splice variants with ASyn (Figure 3C). Alignments of previously-available ESTs predicted two alternative splicing events in ODC1 and three in Asyn. To identify the type of splicing events in each of these splice variants, we have cloned and sequenced all amplified products. The types of alternative splicing events and their influence on the predicted proteins are presented in Figures $3 \mathrm{D}$ and $3 \mathrm{E}$. Complete nucleotide and predicted amino acid sequence of all splice variants for both genes are provided in Supplement 2. Our RT-PCR results show that ODC1 produces more isoforms than predicted from EST alignments, suggesting that the available ESTs/cDNAs because of their limited number, do not predict all alternative splicing events in a gene.

Sequence analysis has revealed that five of the six forms are due to alternative splicing of the 4th intron, which is the largest in this gene. In a study of alternative splicing of SR genes, which undergo extensive alternative splicing in Arabidopsis, it was found that in almost all SR genes the longest intron was involved in generating multiple transcripts by alternative splicing. The alternative splicing events observed in ODC1 include intron retention, Alt5' and Alt3' events. Only one of the six isoforms produced the full-length protein of 542 amino acids, which contains all of the seven conserved signature motifs of ODC1; the remaining five splice variants are predicted to produce three different truncated proteins with 151 to 172 amino acids due to in-frame translation termination codons. None of these three proteins contain conserved regions found in ODC1, hence are not likely to be functional. In humans, splice variants that have a premature termination codon at more than 50 nucleotides upstream of the last 3' exon-exon junction are known to be degraded by NMD [33]. Five of the six splice variants of ODC1 meet this criterion, hence are likely to be the targets of the NMD surveillance system. Interestingly, all five splice variants with a PTC are abundant and in some cases are present in higher levels when compared to the functional transcript (Fig. 3C, compare the lower band to the rest of the bands), suggesting some type of regulatory role for these other transcripts. Three of the four splice variants of the ASyn gene also encode truncated proteins (Figure 3E, Additional File 2). Two of these isoforms (isoform 3 and 4) are also likely targets of NMD. Of the three predicted splice variants for the ASyn gene we verified one of them (isoform 1), and detected a novel splice variant (isoform 2). ASyn transfers the amide group of glutamine to aspartate to form asparagine and plays a role in nitrogen metabolism [42]. Although land plants have 
Table 3 Splice site strength for prevalent and non-prevalent splice forms.

\begin{tabular}{lcccccc}
\hline AS event & \multicolumn{2}{c}{ non-prevalent vs prevalent } & \multicolumn{3}{c}{ prevalent vs constitutive } \\
\hline & non-prevalent avg. score & $p$-value & prevalent avg. score & prevalent avg. score & $p$-value & constitutive avg. score \\
\hline Alt5' & 5.88 & $1.98 \mathrm{e}-07$ & 8.14 & 7.51 & $1.67 \mathrm{e}-07$ & 8.85 \\
\hline Alt3' $^{\prime}$ & 4.17 & $2.30 \mathrm{e}-12$ & 6.73 & 6.31 & $6.95 \mathrm{e}-18$ & 7.57 \\
\hline
\end{tabular}

Splice site scores and $p$-values for the $5^{\prime}$ and $3^{\prime}$ sites of prevalent and non-prevalent splice forms. The table shows data that support two hypotheses: (i) nonprevalent splice sites are weaker than splice sites associated with the prevalent splice form; (ii) prevalent splice sites are weaker than splice sites associated with constitutive splicing. The "avg. score" columns provide the average score of splice site occurrences. For the comparison of non-prevalent with prevalent splice forms, the scores are computed with respect to a motif model of prevalent instances; for the comparison of prevalent and constitutive splicing the scores are computed with respect to a model of the constitutive splice sites.

one or more ASyn genes it is not known if the premRNAs from these undergo alternative splicing [43].

Ornithine decarboxylase is a key rate-limiting enzyme in the biosynthesis of polyamines, which are required for cell growth and cell division in Chlamydomonas and other organisms [44]. It catalyzes the formation of putrescine from ornithine. ODC is present in algae and animals. However, higher plants such as Arabidopsis do not have ODC and synthesize polyamines via a different pathway [45]. ODC pre-mRNA in animals also undergoes alternative splicing. However, the 5 ' untranslated region is alternatively spliced in animals and this event controls ribosomal entry on the ODC mRNA [46]. The physiological significance of ODC1 isoforms in Chlamydomonas remains to be studied. It is possible that the PTC forms may be involved in regulating the level of the functional isoform through regulated unproductive translation and splicing. In addition to these two genes (ODC1 and ASyn) splice variant predictions for a few other genes were verified by others [47-52]. A recent analysis of thylakoid membrane proteins provides support for alternative splicing resulting in the generation of two proteins from a single gene [53].

\section{Conclusions}

During the last six years, the estimates of the extent of alternative splicing in flowering plants has increased from $5 \%$ to $30 \%$ [3] due to an increase in available EST and full-length cDNA sequences. It is likely that the percentage of Chlamydomonas genes known to undergo alternative splicing will increase as more ESTs/cDNAs become available. Deep sequencing of the Chlamydomonas trascriptome under various conditions using next generation sequencing technologies should provide information on the real extent of alternative splicing. However, it is also likely that the prevalence of alternative splicing is roughly four-fold less than in flowering plants. We found that 3\% of Chlamydomonas genes are alternatively spliced; in Arabidopsis, with a similar number of ESTs/cDNAs used in this analysis, about $12 \%$ of genes were predicted to be alternatively spliced [54].

There are only a few cases of alternative splicing reported in Chlamydomonas [47-52]. In several of these the protein coded by splice variants was found to be different, suggesting that proteins generated by alternative splicing may have different functions. In support of this, alternative splicing in some of these genes has been

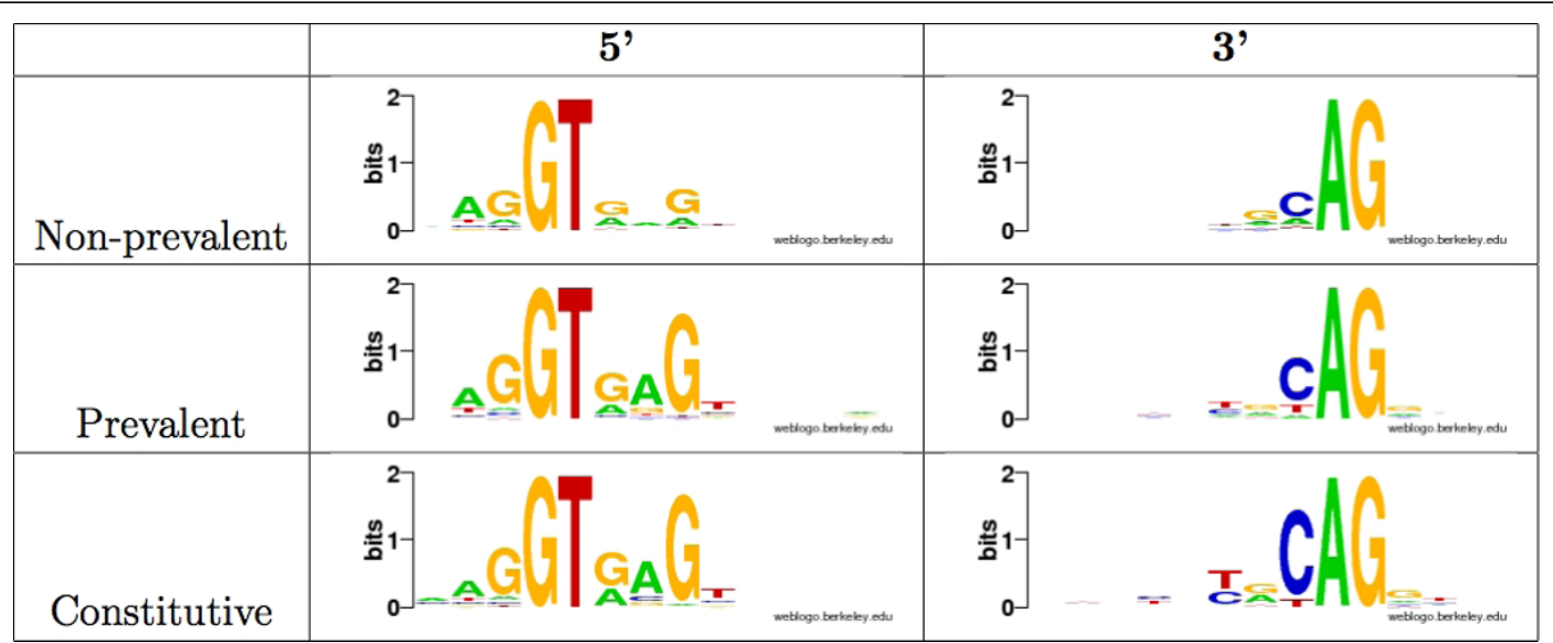

Figure 2 Comparison of splice site motifs for prevalent and non-prevalent splice forms. WebLogo [58] images of $5^{\prime}$ and $3^{\prime}$ splice site motifs for the prevalent and non-prevalent Alt5' and Alt3' splice forms. In the case of Alt5' there is a difference between the prevalent and nonprevalent forms only in the $5^{\prime}$ splice site, and similarly for Alt3', there is a difference only in the $3^{\prime}$ splice site. 
Table 4 The effect of splicing on predicted proteins.

\begin{tabular}{|c|c|c|c|c|c|}
\hline \multirow[b]{3}{*}{$\underline{\text { Event }}$} & \multirow[b]{3}{*}{ \# events } & \multicolumn{2}{|c|}{ AS in Coding Sequence } & \multirow[b]{3}{*}{ AS in UTR \# events } & \multirow[b]{3}{*}{ Tota } \\
\hline & & \multicolumn{2}{|c|}{ ORF Shortened By } & & \\
\hline & & bp & $\%$ & & \\
\hline$\underline{\mathrm{IR}}$ & 30 & 276.52 & $54.52 \%$ & 1 & 31 \\
\hline ES & 4 & 270.00 & $59.74 \%$ & 0 & 4 \\
\hline Alt5' & 6 & 353.62 & $60.67 \%$ & 4 & 11 \\
\hline Alt3' & 22 & 476.32 & $51.37 \%$ & 9 & 31 \\
\hline Total/Avg. & 62 & 359.52 & $54.84 \%$ & 14 & 77 \\
\hline
\end{tabular}

We considered a subset of the clusters with a full-length CDNA, a single alternative splicing event, and a published start codon in the JGI version 4.0 genome annotation. For these clusters we show the number of events in a UTR and the number of events in the coding sequence, where UTRs were detected by the location of where AS occurred with respect to the published start codon and the first stop codon in the reading frame. For events in the coding sequence, we show the average reduction in the length of the predicted ORF that results when comparing the prevalent splice form with the non-prevalent splice form. In all cases but one, the non-prevalent splice form is shorter as a result of a premature termination codon. For IR, the prevalent form is always the one where the intron is spliced, and the non-prevalent form retains the intron. For ES, the prevalent form always contained the exon while the non-prevalent form skipped it.

shown to have a physiological role. Hence, the observed alternative splicing events reported here are likely to be important in regulating gene expression and protein function. Furthermore, alternative splicing may also contribute to the regulation of functional transcript levels through RUST.

Our analysis indicates that alternative splicing is prevalent in Chlamydomonas reinhardtii. However, the extent of alternative splicing is much lower than what is observed in land plants. The frequency of different alternative splicing events is similar to flowering plants, with about half of all splicing events representing intron retention. Our finding that a large number of genes in Chlamydomonas undergo alternative splicing, together with the simplicity of the system and the availability of powerful experimental tools (molecular and genetic) suggest that this organism can serve as an attractive experimental system to understand the mechanisms involved in regulated splicing.

\section{Methods}

\section{Detection of alternative splicing}

To detect potential alternative splicing events, we obtained 252,484 high-fidelity Chlamydomonas EST sequences that Liang et al. corrected using cDNA termini to anchor transcripts to their correct positions in the genome [20]. The ESTs were aligned to the Chlamydomonas genome using the BLAT program [23]. We performed several filtering steps to obtain high quality alignments. The resulting alignments were clustered into putative transcriptional units and processed using a modified version of the Sircah tool to detect alternative splicing events. Details of our alignment processing and alternative splicing detection are found in Additional file 1.

\section{Chlamydomonas Culture}

In our experiments we used two strains of Chlamydomonas reinhardtii (the wall-less strain (cc503) and wt (cc1690)). Both were obtained from the Chlamydomonas Center culture collection at Duke University. These strains were then grown in TAP medium [55]. The cultures were maintained at $22^{\circ} \mathrm{C}$ on a shaking platform in a growth chamber set on a 12:12 light/dark cycle [55]. Cells were subcultured during log phase at a starting density (determined by a hemocytometer) of $10^{5}$ cells/ $\mathrm{mL}$ [55]. For RNA isolation, a $2 \mathrm{ml}$ aliquot was collected during log phase in $2 \mathrm{ml}$ tubes and centrifuged at $0.2 \mathrm{~g}$ for 2 minutes. Supernatant was removed and the procedure repeated until cells from 4 to 6 mls were harvested in the same tube. The resulting pellet was then frozen immediately in liquid $\mathrm{N}_{2}$ and stored at $-20^{\circ} \mathrm{C}$.

\section{RNA Isolation and CDNA synthesis}

Total RNA was isolated using an RNeasy Plant Mini Kit (Qiagen, http://www.qiagen.com/. Prior to RNA isolation, the cell pellet was thawed on ice and frozen in liquid $\mathrm{N}_{2}$. This procedure was repeated 2-3 times in order to lyse the cells and the total RNA was isolated according to the protocol provided by the kit manufacturer. RNA amount was quantified spectrophotometrically at $260 \mathrm{~nm}$. The RNA sample was treated with DNase I according to the manufacturer's instructions (Invitrogen). The quality of RNA was verified by running an aliquot on a $1 \%$ agarose gel. DNase-treated RNA $(1.5 \mu g)$ was used to synthesize first-strand cDNA with an oligo (dT) primer using SuperScriptII (Invitrogen).

\section{PCR of ODC1 and ASyn transcripts}

One-twentieth of the first-strand cDNA was used for PCR amplification in a reaction volume of $20 \mu \mathrm{l}$. The primers were designed using the Primer3 Input http:// frodo.wi.mit.edu/ software. Touchdown PCR (TD-PCR) was performed using a temperature range of $50-60^{\circ} \mathrm{C}$ based upon the primer $\mathrm{T}_{m}$ [56]. An extended hot-start method was utilized in which the PCR sample was 


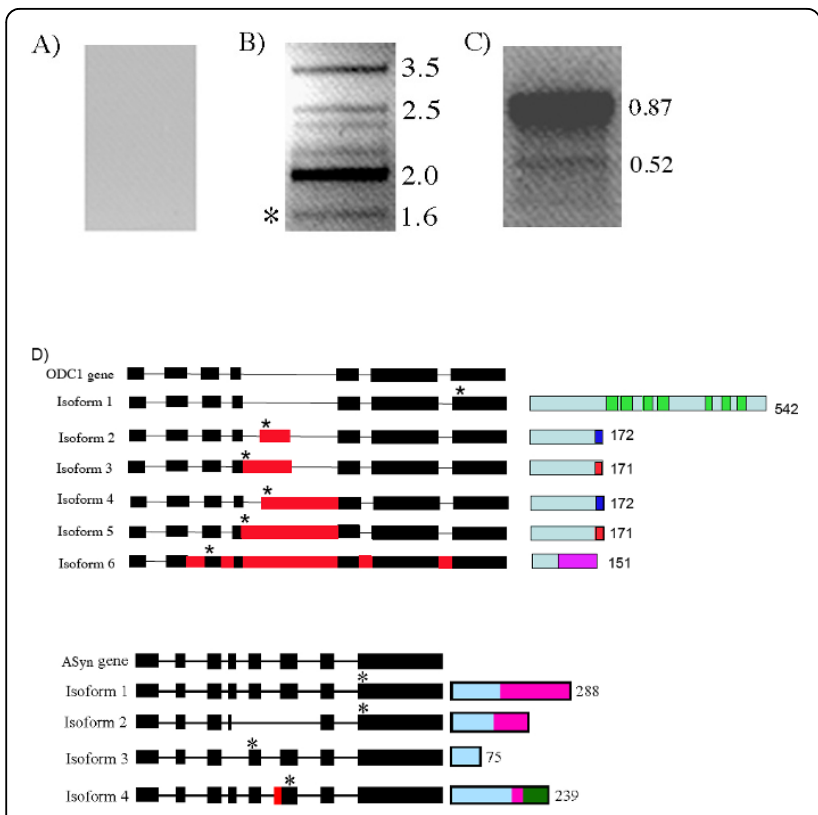

Figure 3 Analysis of ornithine decarboxylase 1 (ODC1) and asparagine synthase (ASyn) splice variants in Chlamydomonas using RT-PCR. A) DNAase-treated RNA was used in PCR with ODC1 primers. B) CDNA amplified with ODC1 primers. An asterisk indicates the spliced form for the full-length protein. Numbers on the right indicate amplified product size in Kb. C) CDNA amplified with ASyn primers. Numbers on the right indicate amplified product size in $\mathrm{Kb}$. D) Diagram showing splicing events in six splice variants (left) and predicted proteins (right) for ODC1. Gene is indicated on top and all six splice variants are shown under the gene. Black boxes indicate constitutively spliced exons and red boxes indicate the included regions in different isoforms. Asterisk indicates the position of translation termination codon. Isoforms 1 to 6 correspond to the bottom to top bands in 3B. The number next to each predicted protein indicates the length of the protein. Conserved signature motifs in ODC1 are represented as green boxes in the full-length protein. Red, blue and magenta colors in truncated proteins represent amino acids unique to them. E) Diagram showing splicing events in four splice variants (left) and predicted proteins (right) for the gene ASyn. The representation of the gene and its splice variants is the same as in D. A conserved domain in ASyn is shown in pink. The green region in isoform 4 represents a unique sequence.

allowed to incubate at $95^{\circ} \mathrm{C}$ for $1.5 \mathrm{hrs}$ prior to PCR cycling. The following TD-PCR conditions were used: initial denaturation performed at $95^{\circ} \mathrm{C}$ for 3 minutes, followed by 10 cycles where denaturation was at $95^{\circ} \mathrm{C}$ for 30 seconds, and an annealing temperature of $60^{\circ} \mathrm{C}$ for 45 seconds. The annealing temperature was set to decrease $0.5^{\circ} \mathrm{C}$ every cycle until the 10 cycles were complete. Elongation was at $72^{\circ} \mathrm{C}$ for 3.5 minutes. The next 20 cycles had a denaturing temperature of $95^{\circ} \mathrm{C}$ for 30 seconds, an annealing temperature of $50^{\circ} \mathrm{C}$ for $45 \mathrm{sec}-$ onds, and an elongation temperature of $72^{\circ} \mathrm{C}$ for 3.5 minutes. The final extension was at $72^{\circ} \mathrm{C}$ for 5 minutes. Amplified PCR products were resolved by electrophoresis in 1\% agarose gels. All PCR reactions were performed using Takara EX Taq ${ }^{\mathrm{Tm}}$ polymerase. Bands were extracted using a razor blade and stored at $-20^{\circ} \mathrm{C}$ until gel extraction was performed.

\section{TOPO Cloning and Sequencing}

Gel extraction was performed prior to TOPO cloning using the GeneJET ${ }^{\text {тм }}$ Gel Extraction kit (Fermentas). After DNA was extracted, the sample was dried using the Speed-Vac and dissolved in $4 \mu l$ of water. The DNA was cloned using the TOPO TA Cloning Kit (Invitrogen). Plasmid from white colonies was isolated using the QIAprep Spin Miniprep kit (Qiagen). Inserts in plasmids were verified using PCR as well as digestion with EcoRI. Clones with an insert were then sequenced at Colorado State Macromolecular Center. Analysis of sequences was performed using the Spidey program [57].

Additional file 1: Supplementary Material. The supplementary material contains additional tables and figures, and a more in-depth description

of the alternative splicing detection and visualization pipeline.

Click here for file

[http://www.biomedcentral.com/content/supplementary/1471-2164-11114-S1.PDF ]

Additional file 2: ODC1 and ASyn sequence information. The file contains complete nucleotide and predicted amino acid sequences of all ODC1 and ASyn splice variants.

Click here for file

[http://www.biomedcentral.com/content/supplementary/1471-2164-11$114-S 2 . D O C]$

\section{Acknowledgements}

This study was funded by NSF award 0743097. We thank Dr. Irene Day for her comments on the manuscript and Jessye Maddox for her help in culturing Chlamydomonas reinhardtii.

\section{Author details}

'Computer Science Department, Colorado State University, Fort Collins, CO, USA. ${ }^{2}$ Department of Biology and Program in Molecular Plant Biology, Colorado State University, Fort Collins, CO, USA.

\section{Authors' contributions}

This study was conceived by $A R$, and designed by $A R$ and $A B$. Bioinformatics analysis was carried out by $A B, A L$, and $M R$, and supervised by $A B$. Wetlab experiments were performed by ALink with the help of JT. AR and $A B$ drafted the manuscript, with contributions from AL, MR, ALink, and JT. All authors read and approved the final manuscript.

Received: 16 June 2009

Accepted: 17 February 2010 Published: 17 February 2010

\section{References}

1. Black DL: mechanisms of alternative pre-messenger RNA splicing. Annual Review of Biochemistry 2003, 72:291-336.

2. Modrek B, Lee C: A genomic view of alternative splicing. Nat Genet 2002, 30:13-19.

3. Reddy A: Alternative splicing of pre-messenger RNAs in plants in the genomic era. Annu Rev Plant Biol 2007, 58:267-294.

4. Barbazuk WB, Fu Y, McGinnis KM: Genome-wide analyses of alternative splicing in plants: Opportunities and challenges. Genome Research 2008, 18(9):1381-1392. 
5. Pan Q, Shai O, Lee L, Frey B, Blencowe B: Deep surveying of alternative splicing complexity in the human transcriptome by high-throughput sequencing. Nature genetics 2008, 40:1413-1415.

6. Lareau L, Brooks A, Soergel D, Meng Q, Brenner S: The coupling of alternative splicing and nonsense-mediated mRNA decay. Advances in experimental medicine and biology 2008, 623:190

7. Lareau L, Inada M, Green R, Wengrod J, Brenner S: Unproductive splicing of SR genes associated with highly conserved and ultraconserved DNA elements. Nature 2007, 446(7138):926.

8. Calarco J, Xing Y, Cáceres M, Calarco J, Xiao X, Pan Q, Lee C, Preuss T, Blencowe B: Global analysis of alternative splicing differences between humans and chimpanzees. Genes \& development 2007, 21(22):2963.

9. Johnson J, Castle J, Garrett-Engele P, Kan Z, Loerch P, Armour C, Santos R, Schadt E, Stoughton R, Shoemaker D: Genome-wide survey of human alternative pre-mRNA splicing with exon junction microarrays. Science 2003, 302(5653):2141-2144.

10. Campbell MA, Haas BJ, Hamilton JP, Mount SM, Buell C: Comprehensive analysis of alternative splicing in rice and comparative analyses with Arabidopsis. BMC Genomics 2006, 7:327.

11. Wang $B B$, Brendel V: Genomewide comparative analysis of alternative splicing in plants. PNAS 2006, 103(18):7175-7180.

12. Palusa S, Ali G, Reddy A: Alternative splicing of pre-mRNAs of Arabidopsis serine/arginine-rich proteins and its regulation by hormones and stresses. Plant J 2007.

13. Tanabe N, Yoshimura K, Kimura A, Yabuta Y, Shigeoka S: Differential expression of alternatively spliced mRNAs of Arabidopsis SR protein homologs, atSR30 and atSR45a, in response to environmental stress. Plant and Cell Physiology 2007, 48(7):1036.

14. lida K, Seki M, Sakurai T, Satou M, Akiyama K, Toyoda T, Konagaya A Shinozaki K: Genome-wide analysis of alternative pre-mRNA splicing in Arabidopsis thaliana based on full-length CDNA sequences. Nucl Acids Res 2004, 32(17):5096-5103.

15. Isshiki M, Tsumoto A, Shimamoto K: The serine/arginine-rich protein family in rice plays important roles in constitutive and alternative splicing of pre-mRNA. Plant Cell 2006, 18:146-158.

16. Ali $G$, Reddy A: Regulation of alternative splicing of pre-mRNAs by stresses. Current topics in microbiology and immunology 2008, 326:257.

17. Kim E, Magen A, Ast G: Different levels of alternative splicing among eukaryotes. Nucl Acids Res 2007, 35:125-131.

18. Merchant S, Prochnik S, Vallon O, Harris E, Karpowicz S, Witman G, Terry A, Salamov A, Fritz-Laylin L, Marechal-Drouard L, other s: The Chlamydomonas genome reveals the evolution of key animal and plant functions. Science 2007, 318(5848):245.

19. Vallon O, Dutcher S: Treasure Hunting in the Chlamydomonas Genome. Genetics 2008, 179:3-6.

20. Liang C, Liu Y, Liu L, Davis A, Shen Y, Li Q: Expressed sequence tags with CDNA termini: previously overlooked resources for gene annotation and transcriptome exploration in Chlamydomonas reinhardtii. Genetics 2008, 179:83.

21. Goodenough U: Green yeast. Cell 1992, 70(4):533.

22. Hu Q, Sommerfeld M, Jarvis E, Ghirardi M, Posewitz M, Seibert M, Darzins A: Microalgal triacylglycerols as feedstocks for biofuel production: perspectives and advances. Plant Journal 2008, 54(4):621.

23. Kent WJ: BLAT - The BLAST-Like Alignment Tool. Genome Research 2002, 12(4):656-664.

24. Harrington E, Bork P: Sircah: a tool for the detection and visualization of alternative transcripts. Bioinformatics 2008, 24(17):1959.

25. Reddy A: Nuclear Pre-mRNA Splicing in Plants. Crit. Rev. Plant Sci 2001, 20:523-571.

26. Lorkovic Z, Wieczorek Kirk D, Lambermon M, Filipowicz W: Pre-mRNA splicing in higher plants. Trends Plant Sci 2000, 5:160-167.

27. Heber S, Alekseyev M, Sze S, Tang H, Pevzner P: Splicing graphs and EST assembly problem. Bioinformatics 2002, 18(Suppl 1):S181-S188.

28. Joint Genome Institute: Chlamydomonas reinhardtii v4.0. 2009http:// genome.jgi-psf.org/Chlre4/Chlre4.download.ftp.html.

29. Zheng CL, Fu XD, Gribskov M: Characteristics and regulatory elements defining constitutive splicing and different modes of alternative splicing in human and mouse. RNA 2005, 11(12):1777-1787.

30. Ner-Gaon H, Leviatan N, Rubin E, Fluhr R: Comparative cross-species alternative splicing in plants. Plant Physiol 2007, 144(3):1632-1641.
31. Lorkovic Z, Kirk D, Klahre U, Hemmings-Mieszczak M, Filipowicz W: RBP45 and RBP47, two oligouridylate-specific hnRNP-like proteins interacting with poly (A)+ RNA in nuclei of plant cells. RNA 2000, 6(11):1610-1624.

32. Maquat L: Nonsense-mediated mRNA decay: splicing, translation and mRNP dynamics. Nat Rev Mol Cell Biol 2004, 5:89-99.

33. Chang YF, Imam JS, Wilkinson ME: The nonsense-mediated decay RNA surveillance pathway. Annual Review of Biochemistry 2007, 76:51-74.

34. Palusa S, Reddy A: Extensive coupling of alternative splicing of premRNAs of serine/arginine (SR) genes with nonsense-mediated decay. The New phytologist 2009, 185:83-89.

35. Kurihara Y, Matsui A, Hanada K, Kawashima M, Ishida J, Morosawa T, Tanaka M, Kaminuma E, Mochizuki Y, Matsushima A, et al: Genome-wide suppression of aberrant mRNA-like noncoding RNAs by NMD in Arabidopsis. Proceedings of the National Academy of Sciences 2009, 106(7):2453.

36. Arciga-Reyes $L$, Wootton $L$, Kieffer M, Davies B: UPF1 is required for nonsense-mediated mRNA decay (NMD) and RNAi in Arabidopsis. Plant $J$ 2006, 47:480-4889.

37. Schoning J, Streitner C, Meyer I, Gao Y, Staiger D: Reciprocal regulation of glycine-rich RNA-binding proteins via an interlocked feedback loop coupling alternative splicing to nonsense-mediated decay in Arabidopsis. Nucleic Acids Research 2008, 36:6977-6987.

38. Long J, Caceres J: The SR protein family of splicing factors: master regulators of gene expression. The Biochemical journal 2009, 417:15.

39. Fairbrother WG, Yeh RF, Sharp PA, Burge CB: Predictive Identification of Exonic Splicing Enhancers in Human Genes. Science 2002, 297(5583):1007-1013.

40. Smith $A$, Sumazin $P$, Zhang M: Identifying tissue-selective transcription factor binding sites in vertebrate promoters. Proceedings of the National Academy of Sciences 2005, 102(5):1560-1565.

41. Majewski J, Ott J: Distribution and Characterization of Regulatory Elements in the Human Genome. Genome Research 2002, 12(12):1827-1836.

42. Inokuchi R, Kuma K, Miyata T, Okada M: Nitrogen-assimilating enzymes in land plants and algae: phylogenic and physiological perspectives. Physiologia Plantarum 2002, 116:1-11.

43. Nakano K, Suzuki T, Hayakawa T, Yamaya T: Organ and cellular localization of asparagine synthetase in rice plants. Plant and Cell Physiology 2000, 41(7):874.

44. Theiss C, Bohley P, Voigt J: Regulation by polyamines of ornithine decarboxylase activity and cell division in the unicellular green alga Chlamydomonas reinhardtii. Plant Physiology 2002, 128(4):1470-1479.

45. Hanfrey C, Sommer S, Mayer M, Burtin D, Michael A: Arabidopsis polyamine biosynthesis: absence of ornithine decarboxylase and the mechanism of arginine decarboxylase activity. Plant Journal 2001, 27(6):551.

46. Pyronnet $S$, Pradayrol L, Sonenberg N: A cell cycle-dependent internal ribosome entry site. Molecular cell 2000, 5(4):607-616.

47. Willmund F, Muhlhaus T, Wojciechowska M, Schroda M: The NH2-terminal domain of the chloroplast GrpE homolog CGE1 is required for dimerization and cochaperone function in vivo. Journal of Biological Chemistry 2007, 282(15):11317.

48. Beligni M, Yamaguchi K, Mayfield S: Chloroplast elongation factor ts proprotein is an evolutionarily conserved fusion with the $s 1$ domaincontaining plastid-specific ribosomal protein-7. The Plant Cell Online 2004, 16(12):3357-3369.

49. Croft M, Moulin M, Webb M, Smith A: Thiamine biosynthesis in algae is regulated by riboswitches. Proceedings of the National Academy of Sciences 2007, 104(52):20770.

50. Falciatore A, Merendino L, Barneche F, Ceol M, Meskauskiene R, Apel K, Rochaix J: The FLP proteins act as regulators of chlorophyll synthesis in response to light and plastid signals in Chlamydomonas. Genes \& development 2005, 19:176-187.

51. Schroda M, Vallon O, Whitelegge J, Beck C, Wollman F: The chloroplastic GrpE homolog of Chlamydomonas: two isoforms generated by differential splicing. The Plant Cell 2001, 13(12):2823.

52. Gonzalez-Ballester D, Pollock S, Pootakham W, Grossman A: The central role of a SNRK2 kinase in sulfur deprivation responses. Plant Physiology 2008, 147:216 
53. Allmer J, Naumann B, Markert C, Zhang M, Hippler M: Mass spectrometric genomic data mining: Novel insights into bioenergetic pathways in Chlamydomonas reinhardtii. Proteomics 2006, 6(23):6207.

54. lida K, Go M: Survey of Conserved Alternative Splicing Events of mRNAs Encoding SR Proteins in Land Plants. Mol Biol Evol 2006, 23:1085-1094.

55. Harris E: The Chlamydomonas Sourcebook, Volume 1: Introduction to Chlamydomonas and Its Laboratory Use Academic Press 2008

56. Korbie D, Mattick J: Touchdown PCR for increased specificity and sensitivity in PCR amplification. Nature Protocols 2008, 3(9):1452-1456.

57. Wheelan S, Church D, Ostell J: Spidey: a tool for mRNA-to-genomic alignments. Genome Research 2001, 11(11):1952-1957.

58. Crooks G, Hon G, Chandonia J, Brenner S: WebLogo: a sequence logo generator. Genome research 2004, 14(6):1188-1190.

doi:10.1186/1471-2164-11-114

Cite this article as: Labadorf et al:: Genome-wide analysis of alternative splicing in Chlamydomonas reinhardtii. BMC Genomics 2010 11:114.

\section{Submit your next manuscript to BioMed Central} and take full advantage of:

- Convenient online submission

- Thorough peer review

- No space constraints or color figure charges

- Immediate publication on acceptance

- Inclusion in PubMed, CAS, Scopus and Google Scholar

- Research which is freely available for redistribution

Submit your manuscript at www.biomedcentral.com/submit
Ciomed Central 